\title{
Computational evaluation of interfacial fracture toughness of thin coatings
}

\author{
M. Bielawski \& K. Chen \\ Institute for Aerospace Research, National Research Council, Canada
}

\begin{abstract}
A computational method to evaluate fracture toughness of single- and multilayered coatings using first-principles density functional theory (DFT) calculations was proposed. This method was first applied to calculate elastic properties and fracture toughness $K_{I C}$ of single crystalline $\mathrm{TiC}$ and several transition metal nitrides with cubic structure, such as $\mathrm{TiN}, \mathrm{CrN}, \mathrm{ZrN}, \mathrm{VN}$ and HfN. After comparison with known experimental data and other DFT results, the reliability of present calculations was favourably confirmed. Next, DFT was applied to calculate the ideal work of adhesion $W_{a d}$, Young's modulus $E$ and interfacial fracture toughness $K_{I C}^{I n t}$ for bi-layer combinations of five transition metal nitrides in (100) and (110) surface orientations. For the analyzed coatings, the following trends were observed: $E(100)>E(110), W_{a d}(100)<W_{a d}(110)$ and $K_{I C}^{I n t}(100)<K_{I C}^{I n t}(110)$, demonstrating that it is the $W_{a d}$ that plays a decisive role in determining interfacial fracture toughness of these materials. All interfaces formed with TiN in the (110) orientation showed the best combination of adhesion and interfacial fracture toughness.
\end{abstract}

Keywords: thin coatings, first-principles calculations, elastic properties, fracture toughness, interfacial fracture toughness.

\section{Introduction}

Erosion-resistant (ER) coatings are used to protect compressor components of land and airborne gas turbines from destructive effects of dust and sand particles suspended in the inflowing air. As particulate dry erosion can significantly impact component life and aircraft safety, attempts were made to develop advanced erosion-resistant coatings for compressor components of gas turbine engines [1-4]. Despite years of effort, high-performing ER coatings capable of 
extending service life of compressor blades by about a factor of ten or more are still sought after. To achieve top performance of erosion-resistant coatings, new materials and new design approaches are being developed [5, 6]. Typically, ER coatings are complex systems, produced using either physical or chemical vapour deposition (PVD or CVD), and consisting of many layers (at the micro and nano scale) of different materials, usually nitrides or carbides of transition metals. The principal damage mechanism for ER coatings is brittle fracture, with crack morphology predominantly controlled by the kinetic energy of impacting particles [7-11]. In the case of small impact velocities, the contact is essentially elastic, and the resulting fracture is in the form of axially symmetric cone cracks starting at the periphery of the contact area. With increased velocities, when plastic deformation occurs, lateral and radial cracks start from the edge of the plastic zone around the impact area. This effect is more pronounced in the case of angular (as oppose to spherical) particles [8]. In actual erosion conditions, under multiple particle impacts, many types of cracks are produced, which leads to material removal when the cracks interlink and propagate to the free surface. In the case of multilayer coatings, developing cracks may reach the interface of individual layers and cause local delamination, leading to an accelerated loss of coating. These mechanisms clearly point to fracture properties as the critical factors in determining erosion resistance of hard and brittle materials [5-9]. In particular, interfacial fracture toughness appears to be one of the key parameters in determining performance of multilayered ER coatings.

Since the traditional methods of developing protective coatings through trialand-error experimentation are time-consuming and costly, extensive modelling and simulation efforts are underway to speed up these developments. However, the materials data needed for the computer models are not easily available. In particular, limited experimental data exist on the fracture toughness of thin films and there are virtually no data on interfacial fracture toughness for thin multilayer coatings. Therefore, developing computational methods that could allow an evaluation of fracture properties of candidate erosion materials would benefit both experimental and modelling efforts.

Density functional theory (DFT) was previously used to calculate elastic constants, electronic structure and adhesion properties of many materials [1218]. Recently, the DFT calculations were extended to evaluation of mechanical properties of structural materials and protective coatings [19-25]. In the present approach, DFT calculations were applied to obtain elastic properties, surface energy and ideal work of adhesion for single crystals of several transition metal nitrides and carbides, leading to the calculation of fracture toughness and interfacial fracture toughness of these materials. To simplify DFT calculations, it was assumed that cracks propagate under Mode I loading in an elastic regime. Since fracture toughness of $\mathrm{TiC}$ and $\mathrm{TiN}$ is relatively well known, these materials were first used to test our approach. Then, DFT calculations were expanded to include directional properties of investigated materials. In this context, the results of DFT calculations of elastic and fracture properties for transition metal nitrides with cubic structure such as $\mathrm{TiN}, \mathrm{CrN}, \mathrm{ZrN}, \mathrm{VN}$ and $\mathrm{HfN}$, and their coherent interfaces in (100) and (110) orientations, are reported. 


\section{Calculation procedure}

The Vienna $a b$ initio simulation package (VASP) [26] using projector augmented wave (PAW) method [27], based on DFT with the generalizedgradient approximation (GGA) [28] was utilized to calculate the total energy $E_{t o t}$ for the investigated cubic structures of TiC, TiN, CrN, ZrN, VN and HfN. From the total energy, the elastic constants $C_{11}, C_{12}$ and $C_{44}$ were derived and Young's modulus $E$ calculated, according to procedure described elsewhere [18].

To obtain Young's modulus along a specific direction, elastic compliances $S_{11}, S_{12}$, and $S_{44}$ were first calculated according to the following formula [29]:

$$
\begin{gathered}
s_{11}=\frac{C_{11}+C_{12}}{\left(C_{11}-C_{12}\right)\left(C_{11}+2 C_{12}\right)} \\
s_{12}=\frac{-C_{12}}{\left(C_{11}-C_{12}\right)\left(C_{11}+2 C_{12}\right)} \\
s_{44}=\frac{1}{C_{44}} .
\end{gathered}
$$

Then, Young's modulus in the direction of unit vector [hkl] was calculated [29]:

$$
\frac{1}{E_{h k l}}=s_{11}-2\left(s_{11}-s_{12}-\frac{1}{2} s_{44}\right)\left(h^{2} k^{2}+k^{2} l^{2}+l^{2} h^{2}\right) .
$$

For elastic coefficient evaluations, the integration in the Brillion zone of $a b$ initio DFT calculations was done using Monkhorst Pack $17 \times 17 \times 17$ k-points of bulk unit cell. For each of the calculations, the Hellman-Feynman forces were calculated to fully relax the atomic coordinates in the models. The planewave cut-off energies were $\sim 450 \mathrm{eV}$ in total energy calculations and the convergence of the total energy calculations was $\sim 10^{-5} \mathrm{eV}$.

Fracture toughness $K_{I C}$, interpreted as a critical stress intensity factor, was calculated from the formula given by Ohring [30]:

$$
K_{I C}=\sqrt{4 \gamma \cdot E},
$$

where $\gamma$ is surface energy calculated from the Boettger formula [31]:

$$
\gamma=\left(E_{\text {slab }}^{N}-N \cdot \Delta E\right) / 2,
$$

where $E^{N}$ slab is the total energy of an $N$-layer slab, and $\Delta E$ the incremental energy determined by $\left(E^{N}{ }_{s l a b}-E^{N-2}\right.$ slab $) / 2$. For these calculations, a series of slab models in (100) orientation and with variable number of layers were generated. It was observed that a critical thickness of 11 layers was enough to achieve the convergence and stable surface energy. In order to prevent interactions between the slab and its periodic images, a vacuum region of at least $1 \mathrm{~nm}$ was included in all supercell models.

Calculations of interfacial fracture toughness $K_{I C}^{\text {Int }}$ were performed using a formula similar to eqn. (5), by substituting surface energy $\gamma$ by interfacial debonding energy $\gamma_{d}$ [30]. Further, for the purpose of this research, the debonding energy of an interface was assumed to be equal to a thermodynamic (ideal) work of adhesion $W_{a d}$ [20]. In addition, to accommodate for interface 
anisotropy, orientation-dependent Young's modulus $E_{h k l}$ was used in these calculations. Thus, the interfacial fracture toughness $K_{I C}{ }^{I n t}$ between two materials along a specific direction $[h k l]$ was calculated from the following formula:

$$
K_{I C}^{I n t}=\sqrt{4 W_{a d} \cdot E_{h k l}} .
$$

Calculations for every pair of materials were performed assuming that the material with the larger Young's modulus was on top of the stack, corresponding to approaching the interface from that side, i.e. giving the upper bound estimation of the interfacial fracture toughness for the given pair of materials.

The work of adhesion $W_{a d}$ was determined through DFT calculations as the difference in total energy between the interface and its isolated surfaces, using the following equation [32]:

$$
W_{a d}=\left(E_{s 1}^{t o t}+E_{s 2}^{t o t}-E_{s 1+s 2}^{t o t}\right) / A,
$$

where $E_{S 1}{ }^{t o t}$ and $E_{S 2}{ }^{t o t}$ are the total energy of slab 1 and slab $2, E_{S I+S 2}{ }^{\text {tot }}$ is the total energy of the interface system consisting of slab 1 and slab 2 separated by the distance $\chi$, and $A$ is the interface area. The interface separation $\chi$, usually expressed in units of lattice constant $a_{0}$, is a parameter in the calculation for the work of adhesion. DFT calculations as per eqn. (8) were performed for a series of $\chi$ distances and the calculated values of $W_{a d}$ were plotted vs. $\chi$ to reveal the minimum, corresponding to the binding (debonding) energy. Two orientations (100) and (110) between nitride pairs have been selected as the interfaces. Since the analyzed nitrides have different lattice parameters, a coherent interface was modelled by assuming an average lattice parameter between the two nitrides [20].

\section{Results and discussions}

DFT calculations were applied to obtain elastic properties for several transition metal nitrides with cubic structure such as TiN, CrN, ZrN, VN and HfN. Table 1 shows the calculated lattice parameters $a_{0}$ and elastic coefficients $C_{11}, C_{12}$ and $C_{44}$ for the selected nitrides. The respective numbers were compared to known theoretical and experimental results for TiN and VN. A good agreement between the current calculations, and other DFT calculations and experimental measurements was observed.

The calculated elastic compliances $S_{11}, S_{12}, S_{44}$ and direction-dependent Young's modulus $E_{h k l}$ for investigated nitrides are listed in Table 2, together with other DFT calculations for TiN and VN. After comparison with literature data, it was found that the current DFT calculations are in good agreement with existing DFT results, thus confirming the reliability of the present approach.

From Table 2, a clear trend of $E(100)>E(110)$ for all five transition metal nitrides can be observed. This trend on Young's modulus indicates that the bond strength in the [100] direction is stronger than that along the [110], which in turn may affect deformation behaviour and mechanical properties of these materials. It is interesting to note that for $E(100), \mathrm{HfN}$ and VN nitrides show larger values than TiN, while in the (110) orientation, it is the TiN that holds the largest value of Young's modulus, indicating that the bond strength of TiN in the (110) 
orientation is stronger compared to that of (100). In both directions, the weakest material in terms of the bond strengths was $\mathrm{CrN}$, as indicated by the lowest $E$ values.

Table 1: $\quad$ Lattice constants $a_{0}$ and elastic coefficients $C_{11}, C_{12}$ and $C_{44}$ for selected nitrides together with other DFT calculations $[15,16]$ and experimental measurements [33, 34].

\begin{tabular}{|c|c|c|c|c|}
\hline & $\begin{array}{c}a_{0} \\
(\AA)\end{array}$ & $\begin{array}{c}C_{11} \\
(\mathrm{GPa})\end{array}$ & $\begin{array}{c}C_{12} \\
(\mathrm{GPa})\end{array}$ & $\begin{array}{c}C_{44} \\
(\mathrm{GPa})\end{array}$ \\
\hline TiN & $\begin{array}{l}4.255,4.27[15] \\
4.26[16], \\
4.26[33]\end{array}$ & $\begin{array}{l}591,604[15] \\
706[16], 625[34]\end{array}$ & $\begin{array}{l}144,136[15] \\
138[16], 165[34]\end{array}$ & $\begin{array}{l}160,162[15] \\
175[16], \\
163[34]\end{array}$ \\
\hline VN & $\begin{array}{l}4.121,4.128[15] \\
4.229[16], \\
4.13[33]\end{array}$ & \begin{tabular}{|l}
$631,636[15]$ \\
$752[16]$, \\
$533[34]$ \\
\end{tabular} & $\begin{array}{l}170,162[15] \\
183[16], \\
135[34]\end{array}$ & $\begin{array}{l}133,126[15] \\
121[16], \\
133[34]\end{array}$ \\
\hline $\mathrm{CrN}$ & 4.124 & 406 & 88 & 75 \\
\hline $\mathrm{ZrN}$ & 4.609 & 583 & 109 & 115 \\
\hline $\mathrm{HfN}$ & 4.530 & 628 & 115 & 120 \\
\hline
\end{tabular}

Table 2: $\quad$ Elastic compliances $S_{11}, S_{12}, S_{44}$ and Young's modulus $E$ in (100) and (110) orientations for selected nitrides along with other DFT calculations [15].

\begin{tabular}{|c|c|c|c|c|c|}
\hline & $\begin{array}{c}S_{11} \\
\left(10^{-12} \mathrm{~m}^{2} \mathrm{~N}^{-1}\right)\end{array}$ & $\begin{array}{c}S_{12} \\
\left(10^{-12} \mathrm{~m}^{2} \mathrm{~N}^{-1}\right)\end{array}$ & $\begin{array}{c}S_{44} \\
\left(10^{-12} \mathrm{~m}^{2} \mathrm{~N}^{-1}\right)\end{array}$ & $\begin{array}{c}E(100) \\
(\mathrm{GPa})\end{array}$ & $\begin{array}{c}E(110) \\
(\mathrm{GPa})\end{array}$ \\
\hline \multirow{2}{*}{$\mathrm{TiN}$} & 1.87 & -0.37 & 6.26 & 534 & 432 \\
& $1.81[15]$ & $-0.3[15]$ & $6.17[15]$ & $554[15]$ & $439[15]$ \\
\hline \multirow{2}{*}{$\mathrm{VN}$} & 1.79 & -0.38 & 7.53 & 559 & 387 \\
& $1.75[15]$ & $-0.4[15]$ & $7.94[15]$ & $570[15]$ & $373[15]$ \\
\hline $\mathrm{CrN}$ & 2.67 & -0.48 & 13.31 & 374 & 226 \\
\hline $\mathrm{ZrN}$ & 1.82 & -0.29 & 8.72 & 548 & 339 \\
\hline $\mathrm{HfN}$ & 1.69 & -0.26 & 8.32 & 593 & 358 \\
\hline
\end{tabular}

The DFT-calculated fracture toughness $K_{I C}$ and surface energy $\gamma$ for TiC, TiN, CrN, ZrN, HfN and VN coatings are listed in Table 3. Again, a very good agreement between the current calculations, other DFT data and experimental measurements was observed, further confirming the reliability of present DFT calculations.

From Table 2 and 3, it can be noted that materials with the largest values of Young's modulus such as HfN and VN at (100) orientation may not necessarily have the largest fracture toughness $K_{I C}$. As HfN shows the largest $K_{I C}(100)$ value of $2.14 \mathrm{MPa} \cdot \mathrm{m}^{1 / 2}, \mathrm{VN}$ is the second lowest with only $1.44 \mathrm{MPa} \cdot \mathrm{m}^{1 / 2}$. A similar example can be found at (110) direction. The important factor here is the surface energy $\gamma$. As indicated by eqn. (5), only coatings with a reasonable combination of $E$ and $\gamma$ can reach high fracture toughness. Not surprisingly, $\mathrm{CrN}$ with the 
lowest $E$ value and very low $\gamma$ (in both directions) yielded the lowest fracture toughness among the investigated materials.

Table 3: $\quad$ Fracture toughness $K_{I C}$ and surface energy $\gamma$ in (100) and (110) orientations for $\mathrm{TiC}$ and selected nitrides with other DFT calculations $[12,13]$ and experimental measurements [35-37].

\begin{tabular}{|c|c|c|c|c|}
\hline & $\begin{array}{c}K_{I C}(100) \\
\left(\mathrm{MPa} \cdot \mathrm{m}^{1 / 2}\right)\end{array}$ & $\begin{array}{c}\gamma(100) \\
\left(\mathrm{J} / \mathrm{m}^{2}\right)\end{array}$ & $\begin{array}{c}K_{I C}(110) \\
\left(\mathrm{MPa} \cdot \mathrm{m}^{1 / 2}\right)\end{array}$ & $\begin{array}{c}\gamma(110) \\
\left(\mathrm{J} / \mathrm{m}^{2}\right)\end{array}$ \\
\hline $\mathrm{TiC}$ & $\begin{array}{c}1.71 \\
1.70[36], \\
1.55[37]\end{array}$ & $\begin{array}{c}1.69 \\
1.67[13],\end{array}$ & - & - \\
& $\begin{array}{c}1.67 \\
\mathrm{TiN}\end{array}$ & $1.71[12]$ & & \\
& $1.76[35]$ & 0.922 & 2.16 & 2.700 \\
\hline $\mathrm{VN}$ & 1.24 & 1.029 & 1.77 & 2.030 \\
\hline $\mathrm{CrN}$ & 1.89 & 1.635 & 1.48 & 1.859 \\
\hline $\mathrm{ZrN}$ & 2.14 & 1.932 & 1.90 & 2.668 \\
\hline $\mathrm{HfN}$ & & & & 2.724 \\
\hline
\end{tabular}

Table 4 lists the calculated ideal work of adhesion $W_{a d}$ and interfacial fracture toughness $K_{I C}{ }^{I n t}$ along the [100] and [110] direction for all 10 coherent interfaces of the five investigated nitrides.

Table 4: $\quad$ Ideal work of adhesion $W_{\text {ad }}$ and interfacial fracture toughness $K_{I C}{ }^{I n t}$ in the (100) and (110) interfaces of selected transition metal nitrides.

\begin{tabular}{|l|c|c|c|c|}
\hline & $\begin{array}{c}\mathrm{W}_{\mathrm{ad}}(100) \\
\left(\mathrm{J} / \mathrm{m}^{2}\right)\end{array}$ & $\begin{array}{c}K_{I C}^{\text {Int }}(100) \\
\left(\mathrm{MPa} \cdot \mathrm{m}^{1 / 2}\right)\end{array}$ & $\begin{array}{c}\mathrm{W}_{\mathrm{ad}}(110) \\
\left(\mathrm{J} / \mathrm{m}^{2}\right)\end{array}$ & $\begin{array}{c}K_{I C}^{\text {Int }}(110) \\
\left(\mathrm{MPa} \cdot \mathrm{m}^{1 / 2}\right)\end{array}$ \\
\hline HfN/TiN & 3.47 & 2.87 & 6.08 & 3.24 \\
\hline VN/TiN & 2.99 & 2.59 & 5.68 & 3.13 \\
\hline ZrN/TiN & 3.23 & 2.66 & 5.84 & 3.18 \\
\hline HfN/VN & 3.11 & 2.71 & 5.55 & 2.93 \\
\hline VN/ZrN & 3.36 & 2.74 & 5.81 & 3.00 \\
\hline HfN/ZrN & 3.49 & 2.88 & 5.82 & 2.89 \\
\hline TiN/CrN & 3.47 & 2.72 & 5.79 & 3.16 \\
\hline VN/CrN & 2.66 & 2.44 & 5.60 & 2.94 \\
\hline ZrN/CrN & 2.93 & 2.54 & 5.52 & 2.74 \\
\hline HfN/CrN & 3.19 & 2.75 & 5.51 & 2.81 \\
\hline
\end{tabular}

Evidently, the work of adhesion values along the [110] direction are larger than those along the [100], i.e. $W_{a d}(110)>W_{a d}(100)$, although Young's moduli (as shown in Table 2) follow the opposite trend $E(100)>E(110)$. Besides, Table 4 shows that the general trend for interfacial fracture toughness follows $K_{I C}^{I n t}(110)>K_{I C}^{I n t}(100)$. Comparison of trends for $K_{I C}^{I n t}$ with those for $E$ indicates that $W_{a d}$ is not fully determined by pure bond strength. Otherwise both 
trends would be identical. It is speculated that the atomic density and packing sequence on that interface may be another factor in controlling $W_{a d}$. Illustration of trends in ideal work of adhesion $W_{a d}$ and interfacial fracture toughness $K_{I C}{ }^{I n t}$ in the (110) direction is shown in Figure 1 for all interfaces formed by the five transition metal nitrides. Similar relationships can be also observed in the (100) direction. Thus, it may be concluded that the ideal work of adhesion $W_{a d}$ plays a decisive role in controlling the interfacial fracture toughness of investigated materials.

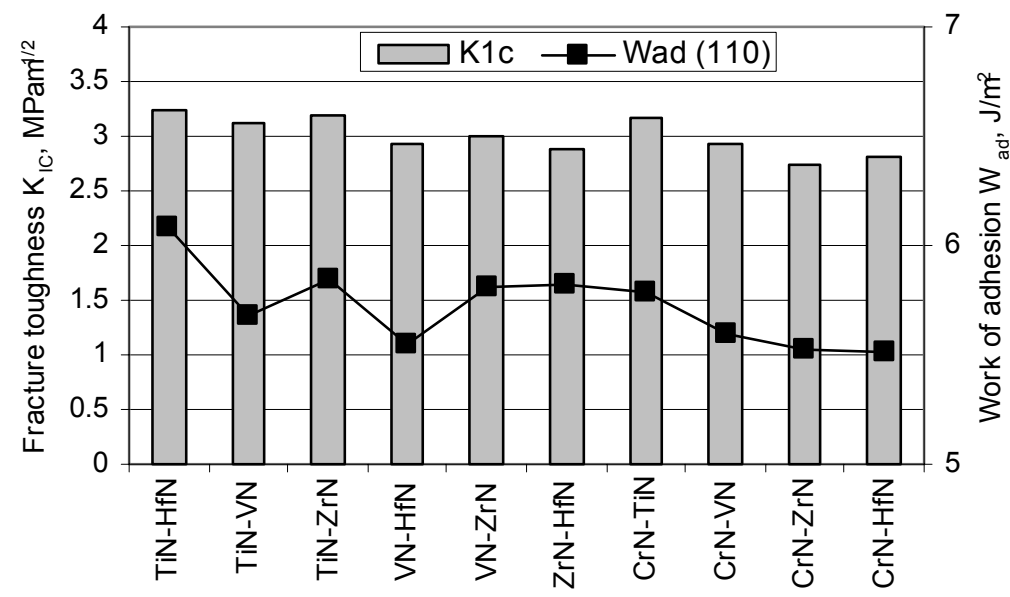

Figure 1: Trends in ideal work of adhesion $W_{a d}$ and interfacial fracture toughness $K_{I C}^{I n t}$ in the (110) interfaces formed by TiN, CrN, VN, $\mathrm{HfN}$ and $\mathrm{ZrN}$.

It should be noticed from Table 4 that in both directions, $W_{a d}$ achieved the largest value on the interfaces of $\mathrm{HfN} / \mathrm{ZrN}, \mathrm{HfN} / \mathrm{TiN}$ and TiN/CrN. However, the largest $K_{I C}{ }^{\text {Int }}$ values were obtained for $\mathrm{HfN} / \mathrm{TiN}, \mathrm{ZrN} / \mathrm{TiN}, \mathrm{TiN} / \mathrm{CrN}$ and $\mathrm{VN} / \mathrm{TiN}$ interfaces in the (110) orientation. Overall, the best combination of adhesion and interfacial fracture toughness was obtained for all interfaces formed with $\mathrm{TiN}$ in the (110) orientation. It can be also noticed from Tables 3 and 4 that on any given orientation, fracture toughness $K_{I C}$ of any nitride is always lower than interfacial fracture toughness $K_{I C}{ }^{I n t}$ of any bi-layer combination. Thus, multi-layered coatings fabricated from these nitrides will have interfaces relatively more resistant to fracture, making them suitable candidates for erosionresistant applications.

\section{Conclusions}

Density functional theory (DFT) calculations have been applied to investigate fracture toughness and interfacial fracture toughness of $\mathrm{TiN}, \mathrm{CrN}, \mathrm{ZrN}, \mathrm{VN}$ and $\mathrm{HfN}$, targeted as erosion-resistant coatings for aerospace applications. DFT 
calculations were performed in (100) and (110) surface orientations. The following conclusions were drawn for the investigated materials.

Only coatings with a reasonable combination of $E$ and $\gamma$ can reach high fracture toughness. The largest fracture toughness was obtained for HfN $\left(2.14 \mathrm{MPa} \cdot \mathrm{m}^{1 / 2}\right)$ in $(100)$ orientation and for TiN $\left(2.16 \mathrm{MPa} \cdot \mathrm{m}^{1 / 2}\right)$ in (110) orientation. The lowest fracture toughness, in both orientations, was obtained for CrN.

Ideal work of adhesion $W_{a d}$ and interfacial fracture toughness $K_{I C}^{I n t}$ along the [100] and [110] direction for all 10 coherent interfaces of the five investigated nitrides were calculated. Overall, the best combination of adhesion and interfacial fracture toughness was obtained for all interfaces formed with TiN in the (110) orientation.

For the analyzed coatings, the following trends were observed: $E(100)>$ $E(110), W_{a d}(100)<W_{a d}(110)$ and $K_{I C}^{I n t}(100)<K_{I C}^{\text {Int }}(110)$, demonstrating that it is the $W_{a d}$ that plays a decisive role in determining interfacial fracture toughness of these materials.

In any given orientation, fracture toughness $K_{I C}$ of any nitride was always lower than interfacial fracture toughness $K_{I C}^{I n t}$ of any bi-layer combination. Thus, multi-layered coatings fabricated from these nitrides will have interfaces more resistant to fracture, making them suitable candidates for erosion-resistant applications.

\section{Acknowledgement}

This work was performed thanks to the New Initiative Fund from the Institute for Aerospace Research of the National Research Council Canada.

\section{Reference}

[1] Paramesvaran, V.R., Immarigeon, J.P. \& Nagy, D., Titanium nitride coating for aero engine compressor gas path components, Surf. Coat. Technol., 52, pp. 251-260, 1992.

[2] Paramesvaran, V.R., Nagy, D., Immarigeon, J.P., Chow, D. \& Morphy, D., Erosion resistant coatings for compressor applications (Chapter 13.0). Advances in High Temperature Structural Materials and Protective Coatings, ed. A.K. Koul, V.R. Paramesvaran, J.P. Immarigeon \& W. Wallace, Publication from National Research Council of Canada: Ottawa, pp. 262-281, 1994.

[3] Tabakoff, W., Protection of coated superalloys from erosion in turbomachinery and other systems exposed to particulate flows, Wear, 233235, pp. 200-208, 1999.

[4] Klein, M. \& Simpson, G., The development of innovative methods for testing a Russian coating on GT T64 gas turbine engine compressor blades, Proc. of the ASME Turbo Expo, CD-ROM peer -reviewed, ASME: Vienna, pp. 1-6, 2004. 
[5] Bielawski, M. \& Beres, W., FE modelling of surface stresses in erosionresistant coatings under single particle impact, Wear, 262, pp. 167-175, 2007.

[6] Hassani, S., Bielawski, M., Beres, W., Martinu, L., Balazinski, M. \& Klemberg-Sapieha, J.E., Design of hard coating architecture for the optimization of erosion resistance, Wear, 265, pp. 879-887, 2008.

[7] Finnie, I., Some reflections on the past and future of erosion, Wear, 186187, pp. 1-10, 1995.

[8] Field, J.E. \& Hutchings, I.M., Surface response to impact (Chapter 7). Materials at High Strain Rates, ed. T.Z. Blazynski, Elsevier: London and New York, pp. 243-293, 1987.

[9] Evans, A.G., Impact damage mechanics: solid projectiles (Chapter 1), Treatise on Materials Science and Technology: Vol. 16 Erosion, ed. C.M. Preece, Academic Press: New York, pp. 1-67, 1979.

[10] Ruff A.W. \& Wiederhorn, S.M., Erosion by solid particle impact (Chapter 2). Treatise on Materials Science and Technology: Vol. 16 Erosion, ed. C.M. Preece, Academic Press: New York, pp. 69-126, 1979.

[11] Gachon, Y., Ienny, P., Forner, A., Farges, G., Sainte Catherine, M.C. \& Vannes, A.B., Erosion by solid particles of W/W-N multilayer coatings obtained by PVD process, Surf. Coat. Technol., 113, pp. 140-148, 1999.

[12] Dudiy, S.V. \& Lundqvist, B.I., First-principles density-functional study of metal-carbonitride interface adhesion: $\mathrm{Co} / \mathrm{TiC}(001)$ and $\mathrm{Co} / \mathrm{TiN}(001)$, Phys. Rev. B, 64, pp. 45403-45417, 2001.

[13] Arya, A. \& Carter, E.A., Structure, bonding, and adhesion at the $\mathrm{TiC}(100) / \mathrm{Fe}(110)$ interface from first principles, J. Chem. Phys., 118, pp. 8982-8996, 2003.

[14] Chen, K., Zhao, L. \& Tse, J., Ab initio study of elastic properties of Ir and $\mathrm{Ir}_{3} \mathrm{X}$ compounds, J. Appl. Phys., 93, pp.2414-, 2003.

[15] Lazar, P., Redinger \& J. Podloucky, R., Density functional theory applied to VN/TiN multilayers, Phys. Rev. B, 76, pp. 174112-174121, 2007.

[16] Wolf, W., Podloucky, R., Antretter, T. \& Fischer, F.D., First-principles study of elastic and thermal properties of refractory carbides and nitrides, Philos. Mag. B, 79, pp. 839-858, 1999.

[17] P. Ravindran, L. Fast, P.A. Korzhavyi, B. Johansson, Density functional theory for calculation of elastic properties of orthorhombic crystals: Application to TiSi 2 , J. Appl. Phys., 84, pp. 4891-4904, 1998.

[18] Chen, K., Zhao, L., Rodgers, J. \& Tse, J.S. Alloying effects on elastic properties of TiN-based nitrides, J. Phys. D: Appl. Phys., 36, pp. $2725-$ 2729. 2003.

[19] Hu, Q.M. \& Yang, R., Mechanical properties of structural materials from first-principles, Curr. Opin. Solid. St. M., 10, pp. 19-25, 2006.

[20] Chen, K. \& Bielawski, M., Interfacial fracture toughness of transition metal nitrides, Surf. Coat. Technol., 203, pp. 598-601, 2008.

[21] Ding, Z., Zhou, S. \& Zhao, Y., Hardness and fracture toughness of brittle materials: A density functional theory study, Phys. Rev. B, 70, pp. $184117-$ 6, 2004. 
[22] Medvedeva, N.I. \& Freeman, A.J., Cleavage fracture in $\mathrm{Ti}_{3} \mathrm{SiC}_{2}$ from firstprinciples, Scripta Mater., 58, pp. 671-674, 2008.

[23] Chen, K. \& Bielawski, M., Ab initio study on fracture toughness of $\mathrm{Ti}_{0.75} \mathrm{X}_{0.25} \mathrm{C}$ ceramics, J. Mater. Sci., 42, pp. 9713-9716, 2007.

[24] Aouadi, S.M., Structural and mechanical properties of TaZrN films: Experimental and ab initio studies, J. Appl. Phys., 99, pp. 53507-6, 2006.

[25] Chen, K. \& Zhao, L., Elastic properties, thermal expansion coefficients and electronic structures of $\mathrm{Ti}_{0.75} \mathrm{X}_{0.25} \mathrm{C}$ carbides, J. Phys. Chem. Solids., 68, pp. 1805-1811, 2007.

[26] Kresse, G., Furthmuller, J., Efficiency of ab-initio total energy calculations for metals and semiconductors using a plane-wave basis set, Comput. Math. Sci., 6, pp. 15-50, 1996.

[27] Kresse, G. \& Joubert, J., From ultrasoft pseudopotentials to the projector augmented-wave method, Phys. Rev. B, 59, pp. 1758-1775. 1999.

[28] Perdew, J.P., Chevary, J.A., Vosko, S.H., Jackson, K.A., Pederson, M.R., Singh, D.J. \& Fiolhais, C., Atoms, molecules, solids, and surfaces: Applications of the generalized gradient approximation for exchange and correlation, Phys. Rev. B, 46, pp. 6671-6687, 1992.

[29] Nye, J. F., Physical Properties of Crystals, Oxford University Press: Oxford, 1993.

[30] Ohring, M., The Materials Science of Thin Films, Academic Press: San Diego, pp. 561-570, 1992.

[31] Boettger, J.C., Nonconvergence of surface energies obtained from thin-film calculations, Phys. Rev. B, 49, pp. 16798-16800, 1994.

[32] Siegel, D.J., Hector Jr., L.G. \& Adams, J.B., First-principle study of metalcarbide/nitride adhesion: Al/VC vs. Al/VN, Acta Materialia, 50, pp. 619631, 2002.

[33] Pearson, W.B., A Handbook of Lattice Spacings and Structures of Metals and Alloys, Pergamon Press: Oxford, 1967.

[34] Kim, J.O., Achenbach, J.D., Mirkarimi, P.B., Shinn, M. \& Barnett, S.A., Elastic constants of single-crystal transition-metal nitride films measured by line-focus acoustic microscopy, J. Appl. Phys., 72, pp. 1805-1811, 1992.

[35] Karimi, A., Wang, Y., Cselle, T. \& Morstein, M., Fracture mechanisms in nanoscale layered hard thin films, Thin Solid Films, 420-421, pp. 275-280, 2002.

[36] Maerky, C., Guillou, M.-O., Henshall, J.L. \& Hooper, R.M., Indentation hardness and fracture toughness in single crystal $\mathrm{TiC}_{0.96}$, Mater. Sci. Eng. A., 209, pp. 329-336, 1996.

[37] Warren, R., Measurement of the fracture properties of brittle solids by hertzian indentation, Acta Metallurgica, 26, pp. 1759-1769. 1978. 